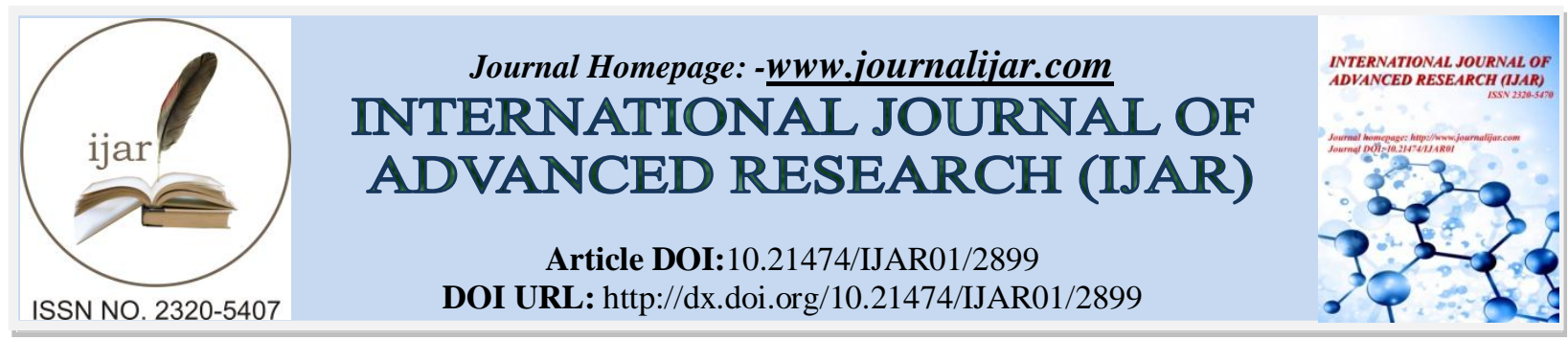

RESEARCH ARTICLE

\title{
EPIDEMIOLOGICAL STUDY OF END STAGE RENAL DISEASE PATIENTS MAINTAINED ON HAEMODIALYSIS IN EL-MINIA GOVERNORATE; EGYPT.
}

\author{
Mohamed Ibrahim Atta ${ }^{1}$, Ashraf Genina ${ }^{2}$, Ashraf Abdel AzeemEwis ${ }^{3}$, Ahmed Amin Ibrahim ${ }^{1}$, \\ NillyHelmyAbdalla ${ }^{1}$ and Warda Sultan Mohamad ${ }^{4}$. \\ 1. Lecturer of Internal Medicine Department, Faculty of Medicine, Beni-Suef University, Egypt. \\ 2. Professor of Internal Medicine Department, Faculty of Medicine, Beni-Suef University, Egypt. \\ 3. Professor of Community Medicine Department, Faculty of Medicine, Minia University, Egypt. \\ 4. M.B.B.CH, MSc internal medicine Faculty of Medicine, Minia University, Egypt.
}

\section{Manuscript Info}

Manuscript History

Received: 23 November 2016

Final Accepted: 25 December 2016

Published: January 2017

Key words:-

ESRD, Epidemiology, Egypt,

Haemodialysis

\begin{abstract}
Objective: The epidemiology of ESRD is important as it determines the need for RRT, a complex, costly and lifelong package of care for which demand and provision have grown significantly in the last decade. Aim of this study is to describe the prevalence and etiology of ESRD in EL-Minia Governorate using a structured questionnaire.

Methods; Epidemiological, clinical and laboratory data of patients with ESRD on regular hemodialysis from all districts of El-Minia governorate were collected on a structured questionnaire.

Results; Out of 1700 ESRD patients on regular HD (314 per million population, pmp), only 1433 patients (84.3\%) agreed to participate in this study, with mean age of $51.6 \pm 13.7$ years. Male patients were $67.8 \%(\mathrm{n}=972), 79.9 \%$ were living in rural areas. Illiterates were $60.6 \%$ of all patients. HTN represents the main cause of ESRD 32.03\%, while unknown etiology represents $26.87 \%$, and $11.4 \%$ DM.

Conclusion; In Egypt, Low prevalence may be due to lack of documentation programs and registration for ESRD and short life expectancy for these patients due to lack of health care
\end{abstract}

Copy Right, IJAR, 2016. All rights reserved.

\section{Introduction: -}

CKD is a major cause of morbidity, mortality, and high medical costs in the United States, particularly among older adults. Nearly 1 in 7 adults has CKD, and recent data suggest that the number of deaths from CKD has doubled in the past 2 decades [1]. In people aged 65 through 74 worldwide, it is estimated that one in five men, and one in four women, have CKD [2]. The lack of community-based screening programs has led to patients being detected with CKD at an advanced stage. It is possible that early detection of kidney disease through community based screening programs might have an impact on this problem through earlier intervention [3]. The epidemiology of ESRD is important as it determines the need for RRT, a complex, costly and lifelong package of care for which demand and provision have grown significantly in the last decade. One year of dialysis costs about $£ 25,000$, the first year of transplantation $£ 15,000$, with subsequent years over $£ 5,000$. It has been estimated that RRT costs consume $1.5-2 \%$ of the NHS budget, a figure that is predicted to rise to at least 3\% [1]. Aim of this study is to describe the prevalence and etiology of ESRD in EL-Minia Governorate using a structured questionnaire. 


\section{Patients and Methods: - \\ Patients: -}

The study included the patients with ESRD on regular hemodialysis in all districts of El-Minia governorate. ElMinia Governorate is one of the Upper Egypt's Governorates, about 234 kilometres to the south of Cairo, it comprises nine districts (fig.1), its population was 5405353 populations according to the records of the Egyptian Ministry of Health and population and the Central Agency of Egypt for public mobilization \& Statistics 2015.

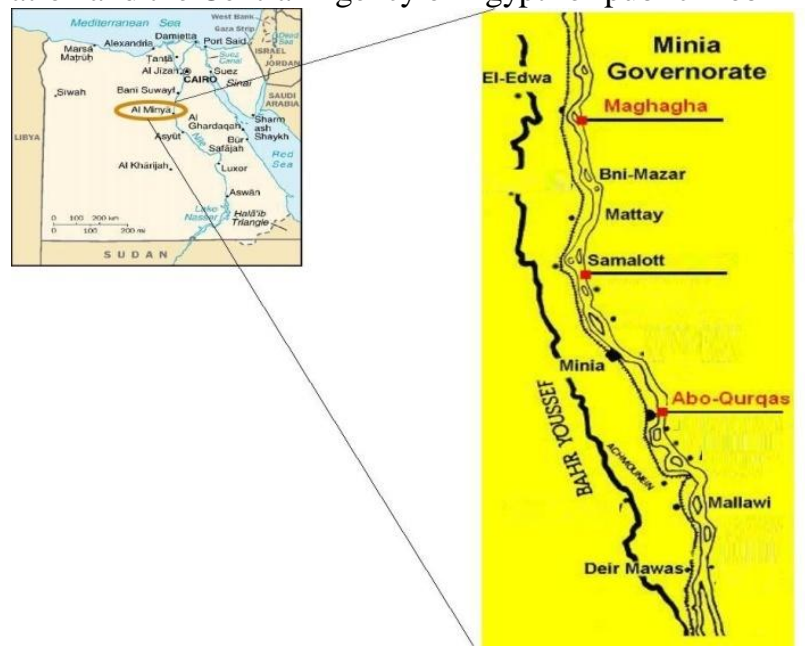

Figure.1: - El-Minia Governorate

The study was conducted between December 2014 to May 2015, the study included 14 dialysis unites, El- Minia University hospital, all general hospitals in the nine districts (El-Edowa general hospital, Maghagha general hospital, Bani-Mazar general hospital, Matti general hospital, Samalout general hospital, El-Minia general hospital, AboKorkas general hospital, Mallawi general hospital, Deir-Mawas general hospital), El-Minia Insurance hospital, ElMinia Fever hospital, and two special centers (Samalout One Day Surgery hospital and Konozecenter).

\section{Methods: -}

Data were collected on a structured questionnaire. The questionnaire included name of the dialysis unit, name of the patient, age, sex, and residence, degree of education, marital status, and their occupation, history of smoking, and the cause of ESRD, history of HCV and HBV infection and if the patient was HCV positive we asked about the time of infection whether it was from the start of the dialysis or not, and asked about risk factor of HCV infection include the following (history of Blood transfusion, going to the dentist, chronic injection, surgeries, relatives infected by $\mathrm{HCV}$, and if the patients deals with other HCV patients, history of bilharziasis and if he was injected with its medication (tartar treatment). Clinical examination was done with special attention to type of vascular access and blood pressure measurement. Blood pressure was measure with a standard sphygmomanometer while the patient was seated, BP measured according to the standared guidelines of the eighth report of the joint national committee (JNC-VIII) on prevention, detection evaluation and treatment of high BP [4]. Hypertension was considered as a cause of ESRD when renal failure was progressive in a patient with long-lasting hypertension, with moderate proteinuria, and no evidence suggestive of another diagnosis. Diabetic nephropathy was diagnosed as history of long-standing diabetes mellitus, with persistent proteinuria, occurrence of diabetic retinopathy and absence of any other renal disease. Diagnosis of chronic glomerulonephritis present on the base of presence of microscopic proteinuria and hematuria with hypertension. Obstructive uropathy was diagnosed by history of being a stone passer and imaging studies. Data obtained from the patients' files included date of the first dialysis session, their laboratory investigations include viral marker (HCV, HBV infection), Urea before dialysis, Urea after dialysis, Creatinine, Hb level, serum iron, Total iron binding capacity (TIBC), serum ferritin, albumin, serum Ca, serum Phosphorus, and parathrmone hormone level, random blood sugar for diabetic patients only, not all patients under go this investigation in dialysis units as it not reported in their files. Data obtained from imaging studies (abdominal ultrasound) the site, size, shape and echogenicity of both kidney, and the stat of the liver, spleen and other organs. Ultrasound can help to identify polycystic kidney disease, cancer, stones, and obstruction. Data obtained about dialysis units: Type of dialysate, type and size of filter. Ethical consideration; the study was conducted after obtaining approvals from the ethical committee of the faculty of medicine Minia University and Beni Suef Faculty of Medicine. Informed consent was obtained from every patient prior to starting filling the questionnaire. 


\section{Statistical methods: -}

Data management; the collected data were coded and entered to the computer. SPSS "Statistical package for social sciences" Version 19 was used for performing the statistical analysis. Qualitative data is presented as frequencies and percentages whereas quantitative data were presented as means and standard deviations. Chi squared test, student $t$ test and other tests of significance were performed whenever needed. P values of less than 0.05 were considered significant.

\section{Results: -}

Out of 1700 ESRD patients on regular HD (314 pmp), only 1433 patients (84.3\%) agreed to participate in this study, their age ranged $13-93$ years with mean age of $51.6 \pm 13.7$ years. Male patients $67.8 \%(\mathrm{n}=972)$ were more than female patients $32.2 \%$ ( $\mathrm{n}=461$ ), $79.9 \%$ of ESRD patients are living in rural areas and $20.1 \%$ are living in urban areas, illiterates were $60.6 \%$ of all patients while $39.4 \%$ were educated (table 1). $14 \%$ were smoker, $24.5 \%$ were exsmokers and $61.5 \%$ were non-smoker. About the marital status of ESRD patients, $10.9 \%$ were single, $76.6 \%$ were married and $11 \%$ were widow. Only $13.6 \%$ of ESRD patients work, and $86.4 \%$ not work (table 1). As regard causes of ESRD, patients with HTN represents $32.03 \%$, unknown etiology were $26.87 \%, 11.4 \%$ DM, $15.14 \%$ were due to obstructive uropathy, $9.14 \%$ due to Glomerulonephritis (GN), $1.39 \%$ were due to Analgesic nephropathy, 1.95\% were due to Polycystic kidney, $0.49 \%$ were due to bilharziasis, 0.49 were due to Systemic Lupus Erythrematosis (SLE) and $1.12 \%$ were due to other causes (obstetric cause and malignancy) (fig. 2). $32.7 \%$ of the patients were in El-Minia city (the capital of El Minia governorate) as it contains 4 units so the main bulk of patients present in it, $12.6 \%$ of the patients were in Abo-Korkas and $12.4 \%$ of the patients were in Mallawi(fig.3). The prevalence of ESRD among different age groups was $8.9 \%$ in those aged < 30 years, $12.1 \%$ in those aged $30-40$ years, $19.3 \%$ in those aged $41-50$ years, $31.8 \%$ in those aged d $51-60$ years, and $27.9 \%$ in those aged $>60$ years. The incidence increased by age and peaked in the 51-60 years age group, slightly decrease after the age of 60 years (table 1). $12.5 \%$ of female patients were less than 30 years, while only $7.2 \%$ of male patients were in the same age group. In female patients, the incidence increase by age, beaked at 41-50 years age group and slightly decrease after the age group 51-60, as regard male patients the incidence increased by the age, peaked in the 51-60 years age group and slightly decrease after the age of 60 years (table 2). 11.1\% from all patients living in urban area were less than 30 years, while $8.4 \%$ from all patients living in rural area were in the same age group, the incidence increased by the age and peaked in the 51-60 years age group, slightly decrease after the age of 60 years and at this age the incidence of the disease were more in rural area (table 2). Table 2 show the highly significant difference (P-value $<0.001 *)$ between patients with different age groups according to their level of education as regard this table the incidence of educated patients with ESRD were decreased progressively with age, while the incidence of illiterate patients with ESRD were increased progressively with age (table 2). In our study, diabetic nephropathy as a cause of ESRD was found to be higher in urban area than rural area (13.2\% vs 10.8\%), also higher in female than male (14.2\% vs 10\%) and higher in illiterate than educated patients (12.8\% vs 9.9\%) (table 3). Obstructive uropathy was higher among male patients $19.6 \%$ with male to female ratio 7:1, polycystic kidney and bilharsiasis higher among male patients $2.1 \%, 0.7 \%$ respectively, analgesic nephropathy was higher in female patients as $2.2 \%$ of female patients develop analgesic nephropathy, also SLE was higher in female patients $1.3 \%$ than male patients (table 3). The incidence of obstructive uropathy, analgesic nephropathy and bilharziasis as a cause of ESRD (16\%, $1.6 \%$ and $0.5 \%$ respectively) were higher in patients living in rural area than those living in urban area, also the incidence of unknown cause was higher in patients living in rural area $28.4 \%$ but the incidence of GN, polycystic kidney, SLE as a cause of ESRD $(13.5 \%, 4.3 \%$, and $1.4 \%$ respectively) were higher in patients living in urban area than those living in rural area (table 3). The incidence of hypertensive nephropathy (34\%), diabetic nephropathy (12.8\%), obstructive uropathy $(15.5 \%)$, analgesic nephropathy $(1.7 \%)$ and bilharsiasis $(0.7 \%)$ were higher in Illiterate patients but the incidence of GN (15.6\%), and SLE (1.1\%) were higher in educated patients (table 3). The incidence of house wife was higher in both areas (81.8\% in urban, and in rural 98.7\%) (table 4). The incidence of don't work male patients were higher in rural area $88.9 \%$, worker patients were higher in urban $43.5 \%$ (table 4). The incidence of lupus nephritis $4.5 \%$, analgesic nephropathy $2.3 \%$ were higher in female patients living in urban area (table 5). The incidence of obstructive uropathy $(20.6 \%)$, analgesic nephropathy $(1.3 \%)$, to bilharziasis $(0.8 \%)$, were higher in male patients living in urban area (table 5). As regard virus status of HD patients in our study, there were 744 patients with positive HCV antibodies (52\%) including 199 were negative at start of HD then seroconverted, while 689 patients were negative to $\mathrm{HCV}$ antibodies (48\%), and only $1.4 \%$ were positive HBsAg (table 6, fig.4). Out of 199 seroconverted patients 164 patients $(82.4 \%)$ were on different frequencies blood transfusion, only 35 patients (17.6\%) never receive blood transfusion and were on erythropoietin and iron replacement therapy, 36.7\% of them under go surgeries and $15.6 \%$ going to dentist (table 7). There was highly significant difference between seroconversion of HCVab+ve and those who remains HCVab-ve as regarding the age and duration of dialysis (P 
value $=0,000$ ) (table 8). In table 9 a correlation test show that seroconverted patients had longer duration of dialysis than other HCV negative patients. There was no statistically significant difference between seroconversion of $\mathrm{HCVab}+\mathrm{ve}$ and those who remains HCVab-ve as regard the gender ( $\mathrm{P}$ value=0.562) (Tab 10). The rate of seroconversion of HCVAb is variable in different districts of ElMinia governorate, the highest in University hospital (44.7\%) and the lowest in Malawi hospital (5.6\%) (Tab 11, Fig 5). Our study showed that a highly significant difference was present among patients received parenteral therapy for Schistosomiasis than those who did not receive parenteral therapy, as $79.5 \%$ of patients who did not receive parenteral therapy was negative for HCV infection, while $51.1 \%$ of those received parenteral therapy for Schistosomiasis were positive for HCV infection (Table 12). Out of $1433 \mathrm{HD}$ patients 20 patients were positive for HBVsAg (1.4\%), 8 (0.6\%)patients were Combined positive for HBVsAg and HCVabs and 12(0.8\%) patients were positive for HBVsAg only (table 13, fig 6). The incidence of hypertensive patients was $67.06 \%$, diabetic patients were $11.6 \%$, cardiac patient were $2.7 \%$ (table 14). In our study diabetes represent $11.4 \%$ of the cause of ESRD, $60.73 \%$ of the diabetic patients were controlled with medical treatment, $38.05 \%$ patients were controlled without medical treatment and $1.22 \%$ was not controlled with medical treatment (table 15). 59.52\% of hypertensive patients were controlled with medical treatment, $43.34 \%$ was not controlled with medical treatment (table 16). In our study number of patients undergoes laboratory investigation widely variant; $\mathrm{Hb}$ level, Creatinine and urea before dialysis were the most common laboratory investigation done to HD patients in El-Minia governorate (table 17). In our study, we found that the mean duration of dialysis was $59.1 \pm 39.7$ months. As regard the vascular access, only $2.6 \%$ of the cases used jugular venous catheter, $0.1 \%$ of the cases used femoral venous catheter during dialysis and $97.3 \%$ of patients used arteriovenous fistula (table 18). As regard type of dialysate solution used for dialysis, 1138 patients (79.4\%) used acetate solution in dialysis while only 295 patients (20.6\%) used bicarbonate solution. Dialysis units of Minia General hospital and Konoz center are the only units that used only bicarbonate solutions for all patients (table 19). As regard Size of filters used for dialysis, 1036 patients (72.3\%) used filters of size $1.3 \mathrm{~m}^{2}$, while only 332 patients (23\%) used filters of size $1.6 \mathrm{~m}^{2}$ (table $\mathbf{2 0}$ ).

Fig2:-Causes of renal failure of the studied group

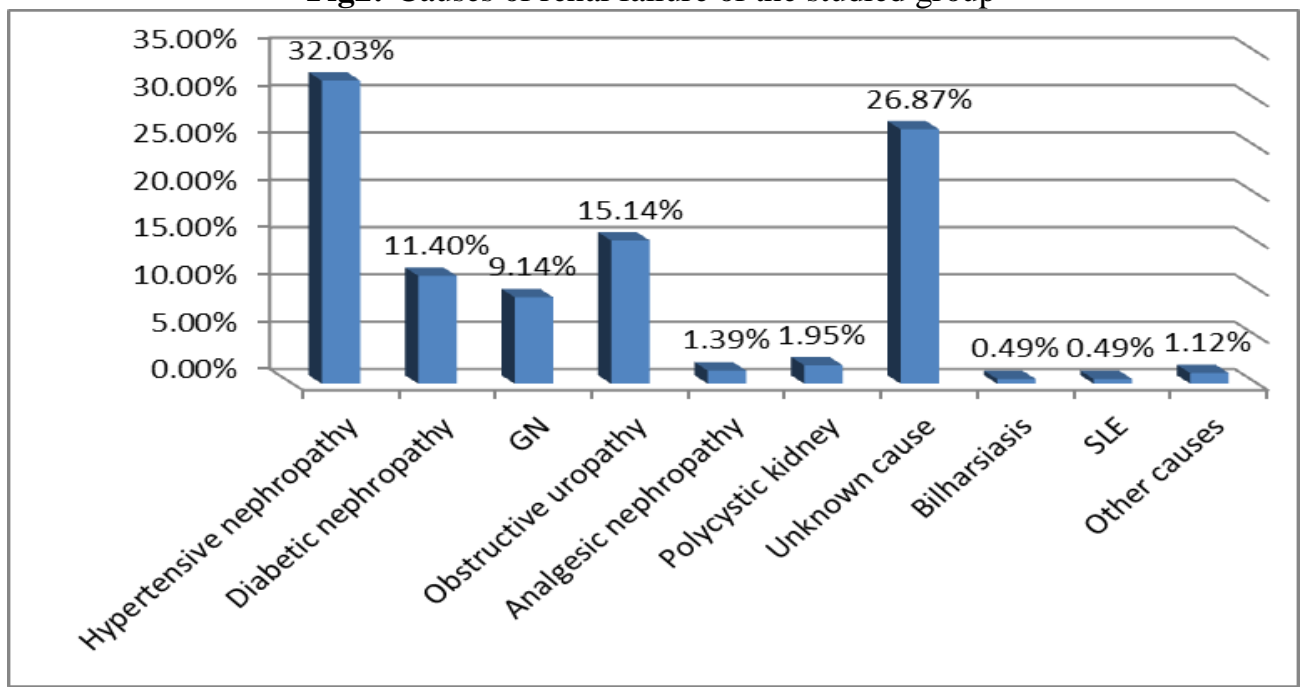


Fig. 3:-Prevalence of ESRD in different districts of Minia Governorate 2015

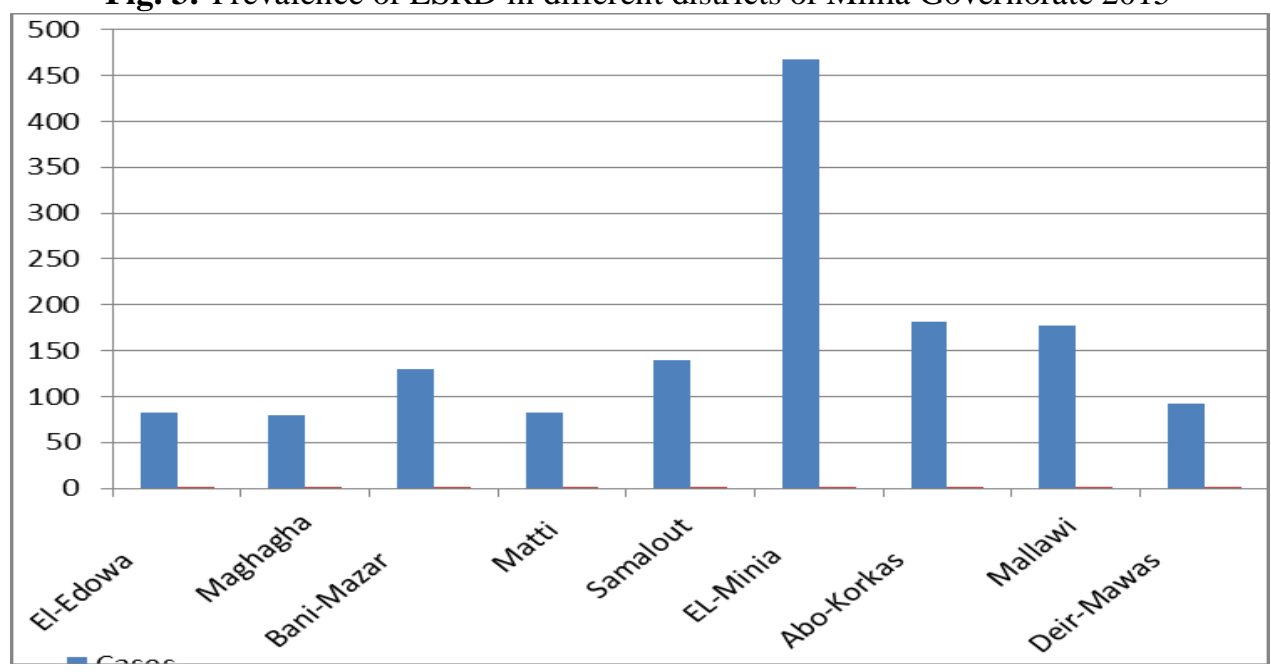

Table 1:-General characteristics of the studied group $(\mathrm{N}=1433)$

\begin{tabular}{|c|c|c|}
\hline \multicolumn{3}{|l|}{ Baseline characteristics } \\
\hline \multirow{2}{*}{ Age (years) } & Range & $13-93$ \\
\hline & Mean \pm SD & $51.6 \pm 13.7$ \\
\hline \multirow[t]{5}{*}{ Age group in(years) } & $<30$ & $128(8.9 \%)$ \\
\hline & $30-40$ & $173(12.1 \%)$ \\
\hline & $41-50$ & $277(19.3 \%)$ \\
\hline & $50-60$ & $456(31.8 \%)$ \\
\hline & $>60$ & $399(27.9 \%)$ \\
\hline \multirow{2}{*}{ Sex } & Male & $971(67.8 \%)$ \\
\hline & Female & $462(32.2 \%)$ \\
\hline \multirow[t]{2}{*}{ Residence } & Urban & $288(20.1 \%)$ \\
\hline & Rural & $1145(79.9 \%)$ \\
\hline \multirow[t]{6}{*}{ Education } & Illiterate & $869(60.6 \%)$ \\
\hline & Primary & $102(7.1 \%)$ \\
\hline & Preparatory & $102(7.1 \%)$ \\
\hline & Secondary & $276(19.3 \%)$ \\
\hline & University & $80(5.6 \%)$ \\
\hline & Higher education & $4(3 \%)$ \\
\hline \multirow[t]{4}{*}{ Marital status } & Single & $156(10.9 \%)$ \\
\hline & Married & $1098(76.6 \%)$ \\
\hline & Divorced & $21(1.5 \%)$ \\
\hline & Widow & $158(11 \%)$ \\
\hline \multirow[t]{7}{*}{ Occupation } & Don't work & $1238(86.5 \%)$ \\
\hline & Employee & $173(12.2 \%)$ \\
\hline & Business owner & $3(0.2 \%)$ \\
\hline & Handicraft & $5(0.3 \%)$ \\
\hline & Farmer & $6(0.4 \%)$ \\
\hline & Professional & $5(0.3 \%)$ \\
\hline & Missing & $3(0.2 \%)$ \\
\hline \multirow[t]{3}{*}{ Smoking } & Smoker & $200(14 \%)$ \\
\hline & Non-smoker & $881(61.5 \%)$ \\
\hline & Ex-smokers & $352(24.6 \%)$ \\
\hline
\end{tabular}


Table 2:- Comparison between different age groups and gender, residence and level of education among patients (N $=1433$ )

\begin{tabular}{|l|l|l|l|l|l|l|}
\hline & Age group & $\mathbf{3 0}$ & $\mathbf{3 0 - 4 0}$ & $\mathbf{4 1 - 5 0}$ & $\mathbf{5 1 - 6 0}$ & $>\mathbf{6 0}$ \\
\hline \multirow{3}{*}{ Gender } & Male N =971 & $70(7.2 \%)$ & $11011.3 \%)$ & $190(19.5 \%)$ & $303(31.2 \%)$ & $299(30.8 \%)$ \\
\cline { 2 - 6 } & Female N=462 & $58(12.5 \%)$ & $63(13.6 \%)$ & $87(31.4 \%)$ & $153(18.8 \%)$ & $100(21.6 \%)$ \\
\hline \multirow{2}{*}{ Residence } & Urban N=288 & $32(11.1 \%)$ & $4(14.2 \%)$ & $55(19.1 \%)$ & $94(32.6 \%)$ & $66(23 \%)$ \\
\cline { 2 - 6 } & Rural N=1145 & $96(8.4 \%)$ & $132(11.5 \%)$ & $222(19.5 \%)$ & $362(31.6 \%)$ & $333(29 \%)$ \\
\hline \multirow{2}{*}{ Education } & Illiterate N=869 & $31(24.2 \%)$ & $68(39.3 \%)$ & $163(58.8 \%)$ & $307(67.3 \%)$ & $300(75.2 \%)$ \\
\cline { 2 - 6 } & Educated N=564 & $97(75.7 \%)$ & $105(60.7 \%)$ & $114(41.1 \%)$ & $149(32.7 \%)$ & $99(24.9 \%)$ \\
\hline
\end{tabular}

Table 3:- Comparison between gender, residence and level of education regarding the etiology of the ESRD.

\begin{tabular}{|l|l|l|l|l|l|l|}
\hline & $\begin{array}{l}\text { Male } \\
\text { N=971 }\end{array}$ & $\begin{array}{l}\text { Female } \\
\mathbf{N = 4 6 2}\end{array}$ & $\begin{array}{l}\text { Urban } \\
\mathbf{N = 2 8 8}\end{array}$ & $\begin{array}{l}\text { Rural } \\
\mathbf{N = 1 1 4 5}\end{array}$ & $\begin{array}{l}\text { Illiterate } \\
\text { N=869 }\end{array}$ & $\begin{array}{l}\text { Educated } \\
\text { N=564 }\end{array}$ \\
\hline $\begin{array}{l}\text { Hypertensive } \\
\text { nephropathy }\end{array}$ & $306(31.5 \%)$ & $153(33.3 \%)$ & $95(33 \%)$ & $364(32 \%)$ & $310(34 \%)$ & $149(26 \%)$ \\
\hline Diabetic nephropathy & $79(10 \%)$ & $66(14.2 \%)$ & $38(13.2 \%)$ & $125(10.8 \%)$ & $109(12.8 \%)$ & $54(9.9 \%)$ \\
\hline GN & $88(9.1 \%)$ & $43(9.4 \%)$ & $39(13.5 \%)$ & $92(8 \%)$ & $43(5 \%)$ & $88(15.6 \%)$ \\
\hline Obstructive Uropathy & $190(19.6 \%)$ & $27(5.8 \%)$ & $34(11.8 \%)$ & $183(16 \%)$ & $135(15.5 \%)$ & $82(14.5 \%)$ \\
\hline Analgesic nephropathy & $10(1 \%)$ & $10(2.2 \%)$ & $2(0.7 \%)$ & $18(1.6 \%)$ & $15(1.7 \%)$ & $5(0.9 \%)$ \\
\hline Polycystic kidney & $20(2.1 \%)$ & $8(1.7 \%)$ & $12(4.3 \%)$ & $16(1.3 \%)$ & $11(1.3 \%)$ & $17(3 \%)$ \\
\hline Unknown cause & $250(25.7 \%)$ & $135(29.3 \%)$ & $59(20.5 \%)$ & $326(28.4 \%)$ & $231(26.6 \%)$ & $326(28.4 \%)$ \\
\hline Bilharsiasis & $7(0.7 \%)$ & $0(0 \%)$ & $1(0.3 \%)$ & $6(0.5 \%)$ & $6(0.7 \%)$ & $1(0.2 \%)$ \\
\hline Others & $2(0.2 \%)$ & $14(3 \%)$ & $4(1.3 \%)$ & $12(1.1 \%)$ & $8(0.92 \%)$ & $8(1.5 \%)$ \\
\hline SLE & $1(0.1 \%)$ & $6(1.3 \%)$ & $4(1.3 \%)$ & $3(0.3 \%)$ & $1(0.1 \%)$ & $6(1.1 \%)$ \\
\hline
\end{tabular}

Table 4:- Occupation per residence in female and male patients

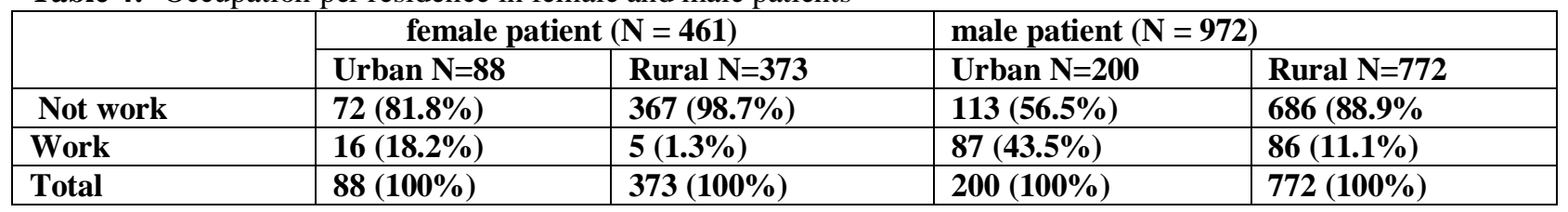

Table 5:-Causes of ESRD according to residence in female and male patient $(\mathrm{N}=461)$

\begin{tabular}{|c|c|c|c|c|}
\hline Etiology & \multicolumn{2}{|c|}{ female patient $(\mathrm{N}=461)$} & \multicolumn{2}{|c|}{ male patient $(\mathrm{N}=972)$} \\
\hline & Urban $\mathrm{N}=\mathbf{8 8}$ & Rural N=373 & Urban $\mathbf{N}=\mathbf{2 0 0}$ & Rural N=772 \\
\hline Hypertensive nephropathy & $30(34.1 \%)$ & $123(33 \%)$ & $65(32.5 \%)$ & $241(31.2 \%)$ \\
\hline Diabetic nephropathy & $12(13.6 \%)$ & $54(14.5 \%)$ & $26(13 \%)$ & $71(9.2 \%)$ \\
\hline GN & $12(13.6 \%)$ & $31(8.3 \%)$ & $27(13.5 \%)$ & $61(7.9 \%)$ \\
\hline Obstructive Uropathy & $3(3.4 \%)$ & $24(6.4 \%)$ & $31(15.5 \%)$ & $159(20.6 \%$ \\
\hline Analgesic nephropathy & $2(2.3 \%)$ & $8(2.1 \%)$ & $\mathbf{0}(\mathbf{0 \%})$ & $10(1.3 \%)$ \\
\hline Polycystic kidney & $3(3.4 \%)$ & $5(1.3 \%)$ & $9(4.5 \%)$ & $11(1.4 \%)$ \\
\hline Unknown cause & $19(21.6 \%)$ & $116(31.1 \%)$ & $40(20 \%)$ & $210(27.2 \%)$ \\
\hline Bilharziasis & $\mathbf{0}(\mathbf{0 \%})$ & $0(0 \%)$ & $1(0.5 \%)$ & $6(0.8 \%)$ \\
\hline Other & $4(4.5 \%)$ & $10(2.7 \%)$ & $1(0.5 \%)$ & $1(0.1 \%)$ \\
\hline SLE & $4(4.5 \%)$ & $2(0.5 \%)$ & $1(0.1 \%)$ & $1(0.1 \%)$ \\
\hline Total & $88(100 \%)$ & $373(100 \%)$ & $200(100 \%)$ & $77(100 \%)$ \\
\hline
\end{tabular}


Fig 4:-Viral status in ESRD patients

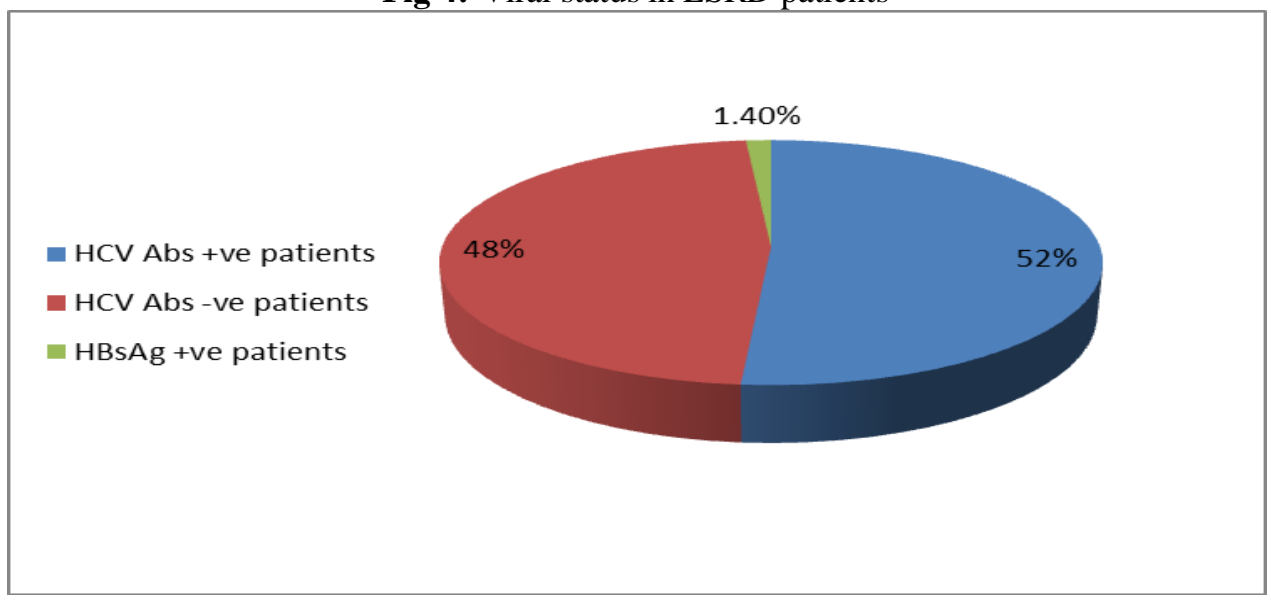

Table 6:- HCV Seroconversion in ESRD patients

\begin{tabular}{|l|l|}
\hline Status & N0 $(\%)$ \\
\hline HCV Abs +ve patients from the start & $\mathbf{5 4 5}(38 \%)$ \\
\hline HCV Abs -ve patients from the start & $\mathbf{8 8 8}(62 \%)$ \\
\hline HCV Seroconverted patients & $199(22.4 \%)$ \\
\hline
\end{tabular}

Table 7:- Risk factor of HCV infection among seroconversion $(\mathrm{N}=199)$

\begin{tabular}{|c|c|c|}
\hline \multicolumn{3}{|c|}{ Risk factor of $\mathrm{HCV}$ among seroconversion } \\
\hline \multirow[t]{2}{*}{ Blood transfusion } & Yes & $164(82.4 \%)$ \\
\hline & No & $35(17.6 \%)$ \\
\hline \multirow[t]{2}{*}{ Going to the dentist } & Yes & $31(15.6 \%)$ \\
\hline & No & $168(84.4 \%)$ \\
\hline \multirow[t]{2}{*}{ Injection } & Yes & $7(3.5 \%)$ \\
\hline & No & $192(96.5 \%)$ \\
\hline \multirow[t]{2}{*}{ Surgeries } & Yes & $73(36.7 \%)$ \\
\hline & No & $126(63.3 \%)$ \\
\hline \multirow[t]{2}{*}{ Relatives infected by HCV } & Yes & $24(12.1 \%)$ \\
\hline & No & $175(87.9 \%)$ \\
\hline \multirow[t]{2}{*}{ Deal with HCV patient } & Yes & $25(12.6 \%)$ \\
\hline & No & $174(87.4 \%)$ \\
\hline
\end{tabular}

Table 8:-Comparison between HCV seroconversion and both the age and duration of dialysis.

\begin{tabular}{|l|l|l|l|l|l|}
\hline \multirow{3}{*}{ Age(months) } & Category & N & Mean & Std. Deviation & P \\
\cline { 2 - 5 } & HCVab -VE & $\mathbf{6 8 9}$ & 49.77 & 14.84 & $\mathbf{0 , 0 0 0}$ \\
\cline { 2 - 5 } & Seroconverted & 199 & 44.3 & 13.14 & \multirow{2}{*}{0,000} \\
\hline \multirow{2}{*}{$\begin{array}{l}\text { Duration of } \\
\text { dialysis(months) }\end{array}$} & HCVab -VE & $\mathbf{6 8 9}$ & $\mathbf{4 7 . 4 4}$ & $\mathbf{2 7 . 4 2}$ & \\
\cline { 2 - 5 } & Seroconverted & 199 & $\mathbf{9 6 . 3 5}$ & $\mathbf{4 8 . 7 6}$ & \\
\hline
\end{tabular}


Table 9:-Correlation between HCV seroconversion and both the age and duration of dialysis.

\begin{tabular}{|c|c|l|l|}
\hline \multicolumn{2}{|c|}{ Correlations } & Age & Duration of dialysis \\
\hline \multirow{2}{*}{$\begin{array}{c}\text { HCV GROUP } \\
\mathbf{n = 8 8 7}\end{array}$} & Spearman's rho (r) & -0.16279 & 0.467 \\
\cline { 2 - 4 } & P value & 0,000 & 0,000 \\
\hline
\end{tabular}

Table 10:-Comparison between HCV seroconversion as regard the gender

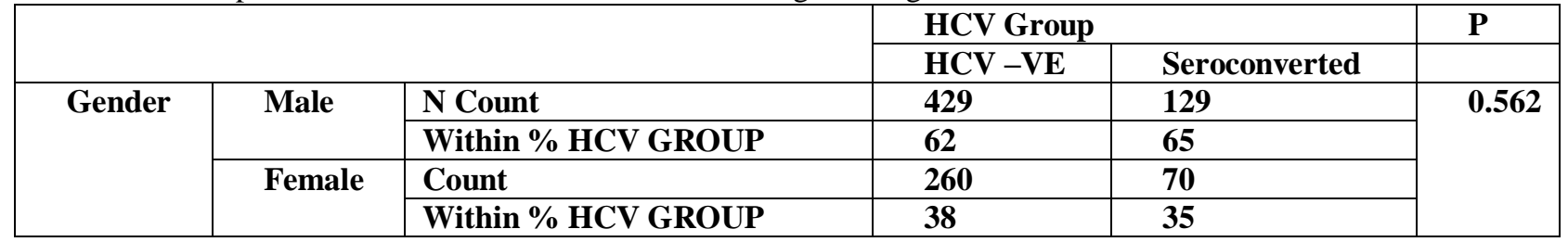

Table 11:- Number and percentage of seroconversion of HCV Ab among different hospitals $(\mathrm{N}=199)$

\begin{tabular}{|c|c|c|c|c|}
\hline Hospital & $\begin{array}{l}\text { Seroce } \\
\text { N } 199 \\
\end{array}$ & rsion & $\begin{array}{l}\text { HCV Ab-ve } \\
\mathrm{N}=689\end{array}$ & $\begin{array}{l}\text { Total HCV Ab-ve before } \\
\text { seroconversion }\end{array}$ \\
\hline Samalout One day surgery hospital & 10 & $40 \%$ & $15(60 \%)$ & 25 \\
\hline Samalout General hospital & 8 & $12.1 \%$ & $58(87.9 \%)$ & 66 \\
\hline Minia University hospital & 42 & $44.7 \%$ & $52(55.3 \%)$ & 94 \\
\hline Konoz center & 7 & $24.1 \%$ & $22(75.9 \%)$ & 29 \\
\hline Health Insurance Hospital & 24 & $27 \%$ & $65(73 \%)$ & 89 \\
\hline Minia General hospital & 27 & $37.5 \%$ & $45(62.5 \%)$ & 72 \\
\hline Mattai General hospital & 12 & $23 \%$ & $40(77 \%)$ & 52 \\
\hline Bani-mazar hospital & 22 & $31.4 \%$ & $48(68.6 \%)$ & 70 \\
\hline Abo-korkas hospital & 11 & $9.2 \%$ & $109(90.8 \%)$ & 120 \\
\hline Maghagha General hospital & 11 & $23.4 \%$ & $36(76.6 \%)$ & 47 \\
\hline El-Edowa hospital & 9 & $18.4 \%$ & $40(81.6 \%)$ & 49 \\
\hline Der-mawas hospital & 9 & $16.4 \%$ & $46(83.6 \%)$ & 55 \\
\hline Malawi hospital & 6 & $5.6 \%$ & $101(94.4 \%)$ & 107 \\
\hline Fever hospital & 1 & $7.7 \%$ & $12(92.3 \%)$ & 13 \\
\hline Total & 199 & $100 \%$ & 689 & 888 \\
\hline
\end{tabular}

Fig 5:-percentage of seroconversion of $\mathrm{HCV} \mathrm{Ab}$ among different hospitals $(\mathrm{N}=199)$

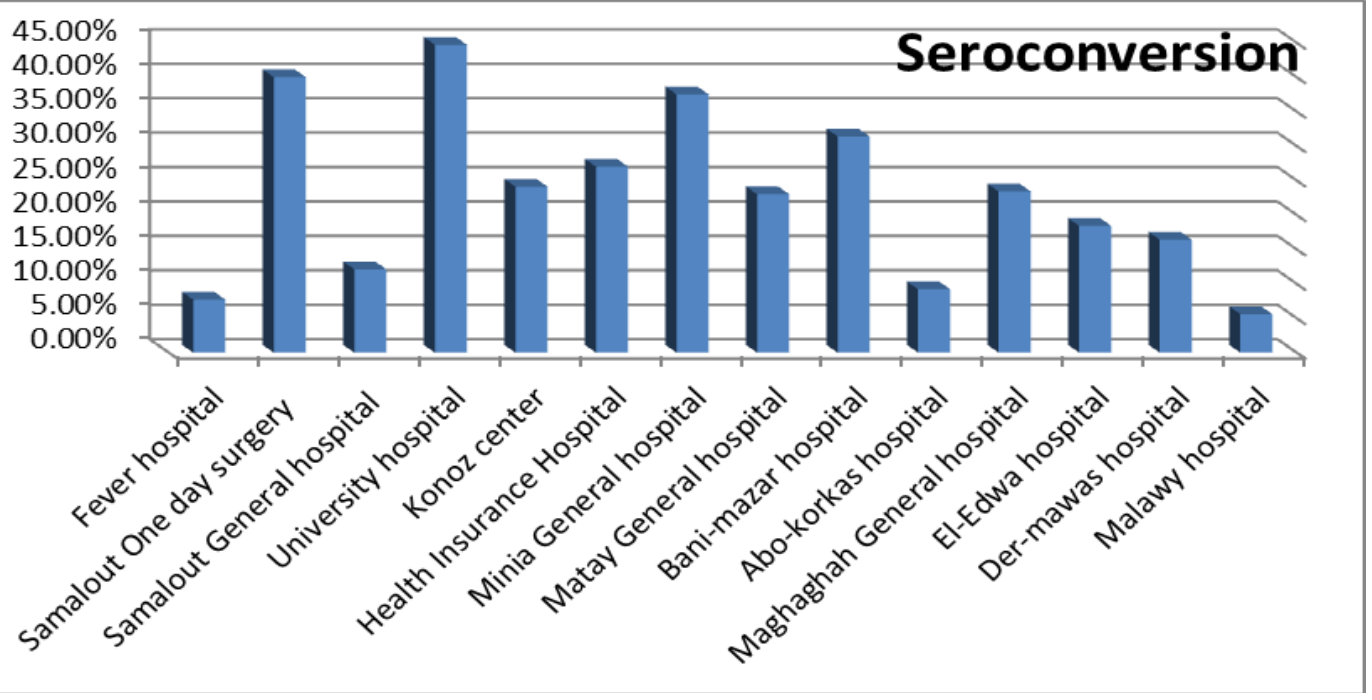


Table 12:-Comparison between HCV +ve and HCV-ve according to parenteral therapy for Schistosomiasis

\begin{tabular}{|c|c|c|c|}
\hline Parenteral therapy for Schistosomiasis & HCV +ve N(\%) & HCV -ve N(\%) & $P$ - value \\
\hline Yes & $364(48.9 \%)$ & $141(20.5 \%)$ & \multirow[t]{3}{*}{$<0.001 *$} \\
\hline No & $380(51.1 \%)$ & $548(79.5 \%)$ & \\
\hline Total & $744(100 \%)$ & $689(100 \%)$ & \\
\hline
\end{tabular}

$*$ Significant difference (p value $\leq 0.05$ )

Table 13:-Classification of patients according to their HBV status

\begin{tabular}{|l|l|}
\hline \multicolumn{2}{|c|}{ HBV infection } \\
\hline HBVsAg +ve patients & $12(0.8 \%)$ \\
\hline Combined HBVsAg +ve and HCV+ve patients & $\mathbf{8}(0.6 \%)$ \\
\hline Total HBVsAg +ve & $20(1.4 \%)$ \\
\hline HBVsAg -ve patient & $1413(98.6 \%)$ \\
\hline Total & $1433(100 \%)$ \\
\hline
\end{tabular}

Fig 6:-HBV status of HD patients

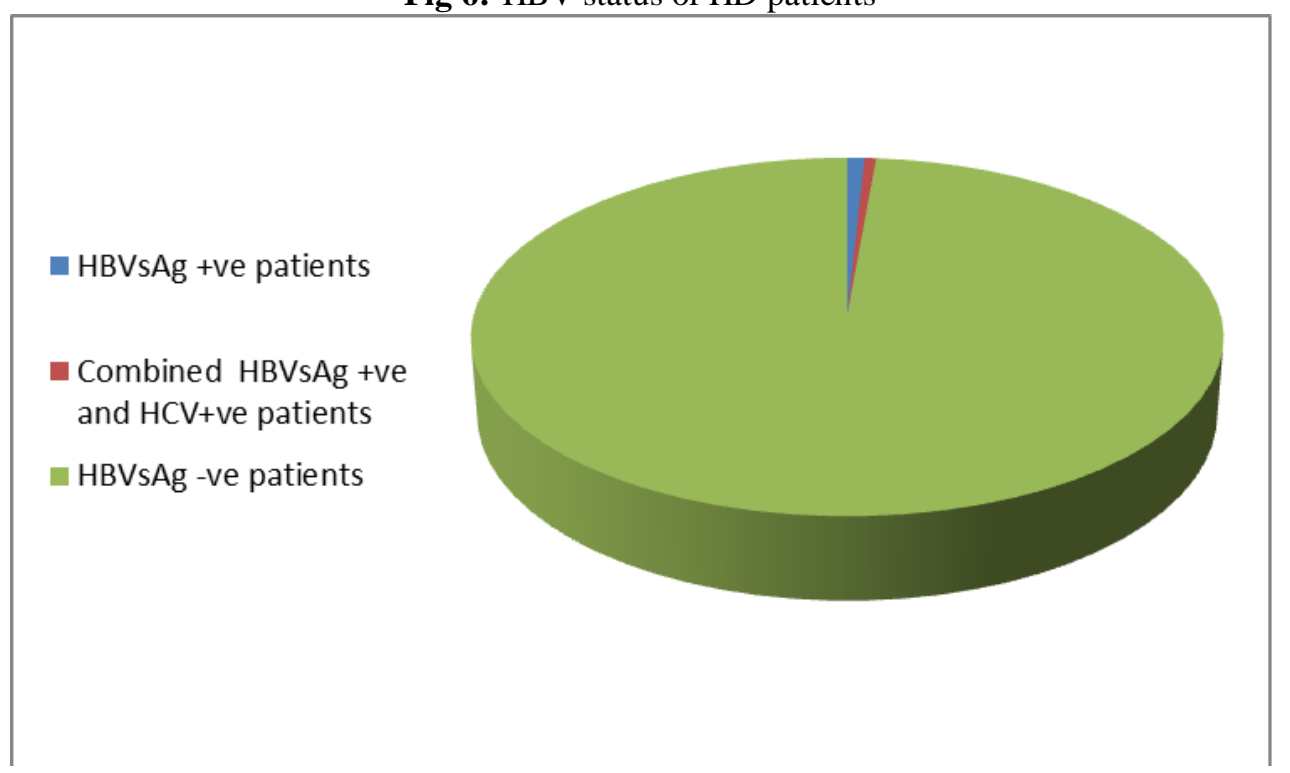

Table 14:-Co-morbidities associated with ESRD

\begin{tabular}{|l|l|l|}
\hline \multirow{5}{*}{ Chronic disease } & No & $430(30 \%)$ \\
\cline { 2 - 3 } & DM & $\mathbf{1 6 3}(11.4 \%)$ \\
\cline { 2 - 3 } & HTN & $\mathbf{9 6 1}(67.06 \%)$ \\
\cline { 2 - 3 } & Cardiac & $\mathbf{3 9}(2.7 \%)$ \\
\cline { 2 - 3 } & Others disease & $\mathbf{9 ( 0 . 6 \% )}$ \\
\hline \multirow{5}{*}{ Bilharziasis } & Yes & $\mathbf{4 8}(3.3 \%)$ \\
\cline { 2 - 3 } & No & $\mathbf{6 4 5}(45 \%)$ \\
\cline { 2 - 3 } & Don't know & $\mathbf{7 4 0}(51.6 \%)$ \\
\hline \multirow{3}{*}{ Back pain } & Yes & $\mathbf{4 6 2}(32.2 \%)$ \\
\cline { 2 - 3 } & No & $\mathbf{9 7 1}(67.8 \%)$ \\
\hline
\end{tabular}

Table 15:- Diabetic control state in HD patients

Diabetic patients $\mathbf{N}=163$

\begin{tabular}{|l|c|}
\hline Controlled with treatment & $99(60.73 \%)$ \\
\hline Controlled without treatment & $\mathbf{6 2}(38.05 \%)$ \\
\hline Not controlled with treatment & $2(1.22 \%)$ \\
\hline
\end{tabular}


Table 16:- HTN control state in HD patients

\section{Hypertensive patients $\mathrm{N}=961$}

Controlled with treatment Controlled without treatment Not Controlled with treatment
$572(59.52 \%)$

$59(6.14 \%)$

$330(34.34 \%)$

Table 17:-Laboratory data of all patients

\begin{tabular}{|c|c|c|c|}
\hline Laboratory data & Number & Range & Mean \pm SD \\
\hline Urea before dialysis & 1365 & $90-320$ & $144.2 \pm 42.8$ \\
\hline Urea after dialysis & 308 & $50-186$ & $70.6 \pm 22.6$ \\
\hline Creatinine & 1364 & $2.5-17.3$ & $7.3 \pm 3.14$ \\
\hline HB & 1432 & $4.2-16$ & $9.5 \pm 1.8$ \\
\hline Ca in blood & 281 & $3.25-11.2$ & $7.7 \pm 1.36$ \\
\hline Phosphorus & 275 & $1.2-10.5$ & $5.87 \pm 1.42$ \\
\hline Parathormon & 280 & $24-5841$ & $95.8 \pm 368.4$ \\
\hline Albumin & 143 & $3-4.5$ & $3.9 \pm 0.29$ \\
\hline Serum iron & 147 & $10-614$ & $83.5 \pm 67$ \\
\hline TIBC & 174 & $11-2645$ & $239 \pm 238$ \\
\hline Feritin & 171 & $11.4-8781$ & $1158 \pm 1422$ \\
\hline
\end{tabular}

Table 18:-Duration and site of dialysis of the studied group $(\mathrm{N}=1433)$

\begin{tabular}{|l|l|l|}
\hline \multirow{2}{*}{ Dialysis } & \\
\hline Duration of dialysis (months) & Range & $\mathbf{7 - 2 8 1}$ \\
\cline { 2 - 3 } & Mean \pm SD & $\mathbf{5 9 . 1} \pm \mathbf{3 9 . 7}$ \\
\hline \multirow{3}{*}{ Vascular access } & A-V shunt & $\mathbf{1 3 9 5 ( \mathbf { 9 7 . 3 } \% )}$ \\
\cline { 2 - 3 } & Jugular catheter & $\mathbf{3 7}(\mathbf{2 . 6 \%})$ \\
\cline { 2 - 3 } & Femoral catheter & $\mathbf{1 ( 0 . 1 \% )}$ \\
\hline
\end{tabular}

N.B: Quantitative data represented by Range (mean \pm SD), While Qualitative data represented by number and (\%).

Table 19:-Solution used for dialysis among different hospitals

\begin{tabular}{|c|c|c|c|}
\hline Hospital & Acetate solution & Bicarbonate solution & Total \\
\hline Samalout One day surgery & $\mathbf{5 4}(\mathbf{1 0 0 \%})$ & - & $\mathbf{5 4}(\mathbf{1 0 0 \%})$ \\
\hline Samalout General hospital & $\mathbf{7 2}(\mathbf{8 3} \%)$ & $\mathbf{1 4}(\mathbf{1 6 \%})$ & $\mathbf{8 6}(\mathbf{1 0 0 \%})$ \\
\hline Minia University hospital & $\mathbf{1 4 6}(\mathbf{1 0 0 \%})$ & - & $\mathbf{1 4 6}(\mathbf{1 0 0 \%})$ \\
\hline Konoz center & - & $\mathbf{3 4}(\mathbf{1 0 0 \%})$ & $\mathbf{3 4}(\mathbf{1 0 0 \%})$ \\
\hline Health Insurance Hospital & $\mathbf{1 5 4}(\mathbf{1 0 0 \%})$ & - & $\mathbf{1 5 4}(\mathbf{1 0 0 \%})$ \\
\hline Minia General hospital & - & $\mathbf{1 1 4}(\mathbf{1 0 0 \%})$ & $\mathbf{1 1 4}(\mathbf{1 0 0 \%})$ \\
\hline Mattai General hospital & $\mathbf{5 4}(\mathbf{6 5 . 1 \%})$ & $\mathbf{2 9}(\mathbf{3 4 . 9 \%})$ & $\mathbf{8 3}(\mathbf{1 0 0 \%})$ \\
\hline Bani-mazar hospital & $\mathbf{9 6}(\mathbf{7 3 . 8 \%})$ & $\mathbf{3 4}(\mathbf{2 6 . 2 \%})$ & $\mathbf{1 3 0}(\mathbf{1 0 0 \%})$ \\
\hline Abo-korkas hospital & $\mathbf{1 5 8}(\mathbf{8 7 . 3 \%})$ & $\mathbf{2 3}(\mathbf{1 2 . 7 \%})$ & $\mathbf{1 8 1}(\mathbf{1 0 0 \%})$ \\
\hline Maghagha General hospital & $\mathbf{7 9}(\mathbf{1 0 0 \%})$ & $\mathbf{7 9}(\mathbf{1 0 0 \%})$ \\
\hline El-Edowa hospital & $\mathbf{7 8}(\mathbf{9 4 \%})$ & $\mathbf{5}(\mathbf{6 \%})$ & $\mathbf{8 3}(\mathbf{1 0 0 \%})$ \\
\hline Der-mawas hospital & $\mathbf{9 2}(\mathbf{1 0 0 \%})$ & $\mathbf{9 2}(\mathbf{1 0 0 \%})$ \\
\hline Malawi hospital & $\mathbf{1 5 4}(\mathbf{8 7 \%})$ & $\mathbf{2 3}(\mathbf{1 3 \%})$ & $\mathbf{1 7 7}(\mathbf{1 0 0 \%})$ \\
\hline Fever hospital & $\mathbf{1 5 \%}(\mathbf{9 5 \%})$ & $\mathbf{2 0}(\mathbf{1 0 0 \%})$ \\
\hline Total & $\mathbf{1 1 3 8}(\mathbf{7 9 . 4 \%})$ & $\mathbf{2 9 5}(\mathbf{2 0 . 6} \%)$ & $\mathbf{1 4 3 3 ( 1 0 0 \% )}$ \\
\hline
\end{tabular}


Table 20:-Size of filters among different hospitals

\begin{tabular}{|c|c|c|c|c|c|c|}
\hline Hospital & $1.2 \mathrm{~m}^{2}$ & $1.3 \mathrm{~m}^{2}$ & $1.4 \mathrm{~m}^{2}$ & $1.6 \mathrm{~m}^{2}$ & $1.8 \mathrm{~m}^{2}$ & Total \\
\hline $\begin{array}{c}\text { Samalout One day } \\
\text { surgery }\end{array}$ & - & $54(100 \%)$ & - & - & - & $54(100 \%)$ \\
\hline $\begin{array}{c}\text { Samalout General } \\
\text { hospital }\end{array}$ & - & $13(15 \%)$ & $\begin{array}{c}27 \\
(31.6 \%)\end{array}$ & $4552.4 \%)$ & - & $86(100 \%)$ \\
\hline Minia University hospital & - & $146(100 \%)$ & - & - & - & $\begin{array}{c}146 \\
(100 \%)\end{array}$ \\
\hline Konoz center & - & $34(100 \%)$ & - & - & - & $34(100 \%)$ \\
\hline $\begin{array}{l}\text { Health Insurance } \\
\text { Hospital }\end{array}$ & - & $154(100 \%)$ & - & - & - & $\begin{array}{c}154 \\
(100 \%)\end{array}$ \\
\hline Minia General hospital & - & $114(100 \%)$ & - & - & - & $\begin{array}{c}114 \\
(100 \%)\end{array}$ \\
\hline Mattai General hospital & - & $83(100 \%)$ & - & - & - & $83(100 \%)$ \\
\hline $\begin{array}{c}\begin{array}{c}\text { Bani mazar General } \\
\text { hospital }\end{array} \\
\end{array}$ & - & - & - & $94(72.3 \%)$ & $36(27.7 \%)$ & $\begin{array}{c}130 \\
(100 \%) \\
\end{array}$ \\
\hline Abo-korkas hospital & $1(0.6 \%)$ & $129(71.3 \%)$ & - & $51(28.2 \%)$ & - & $\begin{array}{c}181 \\
(100 \%) \\
\end{array}$ \\
\hline $\begin{array}{c}\text { Maghagha General } \\
\text { hospital }\end{array}$ & - & $23(29.1 \%)$ & - & $256(70.9 \%)$ & - & $79(100 \%)$ \\
\hline $\begin{array}{c}\text { El-Edowa General } \\
\text { hospital }\end{array}$ & - & $58(69.9 \%)$ & - & $25(30.1 \%)$ & - & $83(100 \%)$ \\
\hline Der mawas hospital & - & $47(51.1 \%)$ & - & $45(48.9 \%)$ & - & $92(100 \%)$ \\
\hline Malawi hospital & - & $161(91 \%)$ & - & $16(9 \%)$ & - & $\begin{array}{c}177 \\
(100 \%)\end{array}$ \\
\hline Fever hospital & - & $20(100 \%)$ & - & - & - & $20(100 \%)$ \\
\hline
\end{tabular}

\section{Discussion: -}

The lack of community-based screening programs has led to patients being detected with CKD at an advanced stage. It is possible that early detection of kidney disease through community based screening programs might improve this problem through earlier treatment [3]. Hemodialysis is the most frequent type of RRT. Mortality is highest during the first 3 months of hemodialysis. Treatment with dialysis or kidney transplantation produces an immense financial burden for many patients who need it [2]. The total cost of the ESRD program in the US was approximately $\$ 49.3$ billion in 2011 . Medicare costs per person per year were more than $\$ 75,000$ overall, ranging from $\$ 32,922$ for transplant patients to $\$ 87,945$ for those receiving hemodialysis therapy [5]. The epidemiology of ESRD is significant as it limits the need for RRT. The rapid increase in the prevalence of ESRD requires practical strategies to prevent its development and progression, especially in the developing world [6]. El-Minia Governorate is one of Egypt Governorates located in the central part of Egypt. In the current study, the total number of patients on RRT were 1700 living in nine districts covering 5.4 million populations only 1433 patients $(84 \%)$ agree to participate in this study, in El-Minia Governorate in 2006 the total number of patients on RRT were 1356 only 800 patients (59\%) agree to participate in the study and in 2007 they were 1615 only 950 patients (59\%) agree to participate in the study [7]. As regard these results the number of ESRD patients was increased annually. The results of our study revealed that in El-Minia Governorate the prevalence of ESRD patients on regular hemodialysis were 314 PMP, this rate was 250 PMP in 2002 [8] and progressively increased to 260 PMP in 2005 [9] then became 308 PMP in 2006 [10], and was 367 pmp in 2007 [7]. In other Egyptian governorates, the prevalence of ESRD patients on regular hemodialysis was $330 \mathrm{pmp}$ in Menoufia Governorate in the year 2013 [11], $282.6 \mathrm{pmp}$ in Kafer El-Shakh Governorate during the year 2012 [12]. The prevalence worldwide was greatly differing. According to USRDS the prevalence was highest in Taiwan with $2447 \mathrm{pmp}$ and was lowest in Philippines with $110 \mathrm{pmp}$. In United States was 1811 pmp[13]. In Europe, the prevalence in 2004 was 760 pmp and increased to 889 pmp in 2008 [14]. In Egypt, Low prevalence may be due to lack of documentation programs and registration for ESRD also short life expectancy for these patients due to lack of health care may play a rule. In the current study $32.7 \%$ of patients on regular HD present in El-Minia city this due to the presence of three large hospitals (university hospital, health insurance hospital, and El- Minia general hospital) in addation to Konozcenter. In the current study, the mean age was $51.6 \pm$ 13.7 but in the year 2006 it was $46 \pm 13$ years [10], this difference may also reflect improvement in the medical services provided for diagnosis and management of ESRD patients which is still much less than in developed 
countries. In France, the median age of patients on RRT is 70.4 years [15]. This marked increase in median age in patients in the European countries may reflect the improvement in ESRD care that requires strong support from the government. The incidence of ESRD in our study increased by age peaked in the 51-60 years age group, and slightly decrease after the age of 60 years. In Bani Suef governorate the mean age was $42.45 \pm 16.2$ years [16], in Menoufia Governorate in the year 2013 the mean age was 52 years [11], and it was 51 years in Kafer El-Shakh Governorate during the year 2012 [12]. In Egypt, the mean age was 45.6 years in 1996 and increased to 49.8 years in 2008 [17]. On studding the patient's residence and its relation to ESRD we find that large number of ESRD patients (79.9\%) live in rural areas and have limited contact to healthcare, only $20.1 \%$ are living in urban areas, also in ElMinia Governorate at the year 2006, 55\% of patients were living in rural areas and $45 \%$ are living in urban areas [10]. In the current study, we found that in the age groups $<30$ years patients living in urban area $(11.1 \%)$ were more than those in rural area (8.4\%) while the reverse occur after the age > 60 as $23 \%$ in urban area and 29\% in rural area this difference in the age groups reflects the difference in awareness of the patients as $75.2 \%$ of the age > 60 years were illiterate. The incidence of obstructive uropathy, analgesic nephropathy, bilharziasis and unknown aetiology as a cause of ESRD was higher in patients living in rural area than those living in urban area. The incidence of hypertensive nephropathy (34\%), diabetic nephropathy (12.8\%), obstructive uropathy (15.5\%), analgesic nephropathy $(1.7 \%)$ and bilharsiasis $(0.7 \%)$ were higher in Illiterate patients but the incidence of GN (15.6\%), and SLE (1.1\%) were higher in educated patients. These differences may reflect the strong link between level of education, living in rural area, health services available and awareness of the patients about the disease status and early referral to nephrologist. In the United Arab Emirates, the highest incidence rate of ESRD was reported in Abu-Dhabi city among the 45-55 years age group [18], while in Kuwait it was 45 years [19]. The incidence of ESRD was more common in male gender worldwide, as USRD System, 2012 revealed that in the US males constituting 56\%, UK, 2009 revealed that males constituting $60 \%$ in the UK, in the KSA males constituting $54.5 \%$ [20]. In our study the prevalence in male was nearly twice that of females $(67.8 \%$ vs $32.2 \%)$. Our results show that only $13.6 \%$ of ESRD patients work, and $86.4 \%$ not work, this reflects the major health problem of ESRD patients because it is a devastating medical condition, and the cost of treatment is a huge economic burden. In the current study the incidence of ESRD of unknown etiology was 26.87\%, and during the year 2006 was 27\% [9], in Bani Suef governorate was 42.2\% [16], in Menoufia Governorate in the year 2013 was $20.5 \%$ of all causes of ESRD [11], and it was $15.2 \%$ in all Egypt [21]. In Sudan, uncertain etiology of ESRD was estimated to be more than $40 \%$ [22]. While in the US it was only 3.9\% [13]. In Saudi Arabia it was $19.9 \%$ [23]. The incidence of ESRD due to unknown etiology in Egypt is like that in Saudi Arabia, but so much higher than that in the US. This difference may be attributed to lake of awareness of the patients, delay referral to nephrologist in developing countries and reduced health care system. In the current study, hypertensive nephropathy was the first leading cause of ESRD in El-Minia Governorate and the incidence was 32.03\%, while it was 27\% in the year 2002 [8], $20 \%$ in the year 2006 [10]. In Bani Suef governorate hypertension represented 15.2\% [16]. In Menoufia Governorate in the year 2013 hypertension $(31.1 \%$ ) was the main cause of ESRD [11], also the main cause of ESRD in Cairo was hypertension (29.7\%), followed by DN (12.5\%), in Canal governorates it was 27.3\%, followed by DN (10.7\%) [12]. In Tabuk Saudi Arabia 24\% of patients were hypertensive [24]. In Sudan hypertension was responsible for about 26\% [22]. Similarly, In US it was 28\% [13]. But this percentage of HTN may be not accurate as a cause of ESRD as it is difficult to decide if long-standing hypertension is the cause of ESRD or a previous undiagnosed renal disease is the cause of secondary HTN. In our study the prevalence of hypertension was higher in females $33.3 \%$ than in males $31.5 \%$. In our study the incidence of diabetic nephropathy was $11.3 \%$, it was $5 \%$ during the year 2005 [8], $8 \%$ during the year of 2006 [10], and 13\% in the year of 2007 [7]. In Bani Suef governorate it was 13.2\% [16]. The prevalence of diabetic nephropathy was higher in females $14.2 \%$ than in males $10 \%$, and more in urban $13.2 \%$ than in rural 10.8\%, this agree with [7] in 2007 diabetic nephropathy was higher in urban (8\%) than in rural areas (5\%), also in Emarate prevalence of diabetic nephropathy appears to be higher in urban and more developed areas than rural areas [18]. In Menoufia Governorate in the year 2013 diabetic nephropathy was $15.9 \%$ [11], 14\% in Kafer ElShakh Governorate during the year 2012 [12]. The prevalence of DN in Egypt as a cause of ESRD was 8.9\% in 1997 and increased to $13.5 \%$ in 2008 and accounting the 2nd cause of ESRD as the main cause is hypertension 36.6\% [21]. In South Africa, it was 14\% to 16\%, and in Sudan it was 9\% [25]. In France, during the year 2007, 39\% of patients with ESRD on HD were diabetics [15]. According to the 2008 United States Renal Data System annual report the major cause of ESRD leading to kidney failure in the US was diabetes [26], 28.9\% of patients on RRT while in Iran diabetes mellitus constitutes $30.1 \%$ of ESRD patients [27]. In Gulf countries like Qatar, diabetic nephropathy was the commonest cause of ESRD 48\% [28], and in Saudi Arabia it was $25.2 \%$ [23]. In France in year 2007, $39 \%$ of ESRD on regular hemodialysis was due to DN [15]. In United Kingdom DN accounts $14.7 \%$ the 2nd cause of ESRD following GN 16\% [29]. This difference in prevalence of DN as a cause of ESRD may be due to the low prevalence of obesity in the less developed areas compared to that in the developed regions because of their 
rapid economic growth and associated changes in lifestyle. Chronic glomerulonephritis was seen in $9.14 \%$ in the current study, in 2006 it represented $11 \%$ of ESRD patients [10], while it was only $1 \%$ in the US. In our study hepatitis C prevalence was found to be $52 \%$, In Menoufia Governorate in the year 2013 hepatitis C prevalence was $38.6 \%$ [11], and was 39.7\% in the Kafer El-Shakh Governorate during the year 2012 [12]. In Bani Suef governorate was $55.79 \%$ [16]. There was a wide variation in hepatitis $\mathrm{C}$ prevalence in dialysis patients present worldwide. It was 52\% in Egypt [21], 54.4 \% in Syria [30], $21 \%$ in Jordan [31], and in Saudi Arabia $18.9 \%$ [32]. Our study showed that $51.1 \%$ of patients received parenteral therapy for Schistosomiasis were positive for HCV infection, this is because previous parenteral therapy for Schistosomiasis without adequate sterilization techniques has been concerned as the cause for this high prevalence rate of hepatitis C in Egypt. Hepatitis C prevalence was high in ElMinia Governorate may be credited to the high hepatitis $\mathrm{C}$ prevalence in the general population, also blood transfusion used by high rate in dialysis units to treat anemia instead of erythropoietin and iron therapy, as it is costly, and lack of infection control standard methods in dialysis units. In our study, HCV seroconversion rate was $22.4 \%$ among patients who were HCV-free at the start of the study, while it was $14.5 \%$ in Menoufia governorate [21], and in Bani Suef governorate was 7.33\% [16]. In the current study the duration of dialysis was significantly high (P-value 0.0001) in seroconverted patients than those remain HCV ab negative, this agree with a study in Bani Suef governorate as the duration of dialysis was significantly high in HCV seroconverted patients [16], in Menoufia governorate Zahran, 2014 showed that the duration of dialysis was the predictor of HCV seroconversion [34]. Also, in India a study demonstrated that the duration of haemodialysis was the important risk factors for HCV seroconversion [35]. Okuda and Hayashi, 1996 showed that 31.6\% were seroconverted at a large dialysis unit in USA a study on 152 patients who had not received blood, and seroconversion was correlated with the dialysis duration [36]. In our study the age was a highly significant risk factor for seroconversion as age decreased incidence of seroconversion increased, the study of Selm, $\mathbf{2 0 1 0}$ in Yemen showed that old age was significant risk factor for seroconversion [37], while in Bani Suef governorate age was insignificant risk factor for seroconversion [16]. Our results agree with a study in Bani Suef governorate that gender was insignificant risk factor for seroconversion [16], while in Yemenmale gender was significant risk factors for HCV seroconversion [37].

\section{Recommendations:-}

As a significant proportion of ESRD patients discovered their kidney problem very late and were not well equipped for renal replacement therapy with a high prevalence of hepatitis $\mathrm{C}$. We recommend the following; first, education program for physicians should be strengthened with special emphasis on etiological factors causing ESRD, increase awareness of primary health care physician and treating physician about the proper time for referral to the nephrologists and increase awareness of nephrologists about the proper time for preparing patient for renal replacement therapy. Statistical evaluation and patient registry of ESRD patients for each governorate is useful to explain the characteristics of ESRD patients and dialysis therapy, to assess complications on the scientific evidence basis, for improving quality of dialysis therapy and to provide information about socioeconomic health administration for a future health plan. Another study for assessment of dialysis adequacy, anemic state of the ESRD patients would be practical and beneficial in providing safe and cost-effective HD treatment. Also, to improve the quality of dialysis therapy all laboratory investigation must be regularly evaluated to all ESRD patients. Proper control of hypertension and follow-up of diabetic patients and annual registry of diabetics on HD. Using of iron therapy and erythropoietin instead of the high rate of blood transfusion to treat anemia in dialysis units and proper use of guidelines for infection control in dialysis units to prevent HCV Seroconversion.

\section{References:-}

1. Hoerger T. J., Sean A., Simpson M.A., Benjamin O et al (2015): The Future Burden of CKD in the United States: A Simulation Model for the CDC CKD Initiative. Am J Kidney Dis.;65(3):403-411

2. National Kidney Foundation(2015): Global Facts: About Kidney Disease,

3. Singh A, Youssef M, Bharati V, Kuyilan K. et al(2013): Epidemiology and risk factors of chronic kidney disease in India results from the SEEK (Screening and Early Evaluation of Kidney Disease) study BioMed Central Nephrology (BMC), 14:114

4. Kayce Bell, June Twiggs, Bernie R. (2015): Hypertension The Silent Killer: Updated JNC-8 Guideline Recommendations Alabama pharmacy ASSOCIATION 2 | Summer: Continuing EDUCATION

5. Gregorio T Obrador, MD, Brian JG et al.,(2014): Epidemiology of chronic kidney disease Topic 7236 Version 16.0 Official reprint from UpTo Date.

6. Aghighi M., Mahdavi MM, Zamyadi M, Heidary A R, et al (2009) :Changing Epidemiology of End-Stage Renal Disease in Last 10 Years in Iran, Iranian Journal of Kidney Disease,(IJKD) 2009;3:192-6 
7. El-Minshawy O. (2011): End Stage Renal Disease in El-Minia Governorate, Egypt: Data of the Year 2007. Nephro-Urol Mon.;3(2):118-121.

8. El Minshawy O, El Sharqawy M, Ewis A. (2002): End stage renal disease in El Minia Governorate (Central Egypt): An epidemiological Study. J Egypt SocNephrol; 4(1):34-41.

9. El Minshawy $O$ and Kamel EG.(2006): Renal replacement therapy and increased risk of cardiovascular disease in El-Minia Governorate, Upper Egypt. Egypt J Hosp Med; 22:29-38..

10. El Minshawy O.(2011): End-Stage Renal Disease in the El-Minia Governorate, Upper Egypt: An Epidemiological Study Saudi J Kidney Dis Transpl; 22(5):10481054

11. 11-Ahmed R, Mahmoud K, Hany S, Hala G and Ahmed S.(2015): Prevalence of End Stage Renal Disease in Menoufia Governorate. Nature and Science Nat Sci;13(6):154-158

12. Hassan A. Ahmeda, Yassine S. Yassinea, Ahmed R. Tawafea, Mohammed M. Ebazawayb(2015): Egypt, end-stage renal disease, epidemiology of haemodialysis, etiology of end-stage renal disease, Kafer El-Shakh Governorate Menoufia Medical Journal, 28:267-271.

13. U S Renal Data System, USRDS (2011): Annual Data Report Atlas of Chronic Kidney Disease and EndStage Renal Disease in the United States, National Institutes of Health, National Institute of Diabetes and Digestive and Kidney Diseases, Bethesda, MD.

14. Stel VS, van de Luijtgaarden MW, Wanner C, Jager KJ(2011): On behalf of the European Renal Registry Investigators, the 2008 ERA-EDTA Registry Annual Report-a précis. NDT Plus. Feb; 4(1):1-13.

15. Couchoud C, Lassalle M, Stengel B, Jacquelinet C.(2009): Renal Epidemiology and Information Network: annual report Nephrol Ther;5 Suppl 1:S3-144.

16. Genina A M, Ebrahim M, Mohamed Z, (2012): African Journal of Nephrology,Prevalence of hepatitis C virus antibodies in end stage renal disease patients in Beni-Suif/Egypt16:132-139.

17. Afifi A.(2015): Egyptian Renal Registry 9th Annual Report.

18. Hassanien A, Al-Shaikh F , Eszter P. et al (2012): Epidemiology of end-stage renal disease in the countries of the Gulf Cooperation Council; J R Soc Med Sh R 2012;3:38. DOI 10.1258/shorts.2012.011150.

19. El-Reshaid K, Johny KV, Sugathan TN, et al. (1994): End-stage renal disease and renal replacement therapy in Kuwaitepidemiological profile over the past 412 years. Nephrol Dial Transplant;9:532-8.

20. Al-Sayyari AA, Shaheen FA. (2011): End Stage Chronic Kidney disease. A rapidly changing scene. Saudi Med J;32:339-46.

21. Zahran A.(2011): Epidemiology of hemodialysis patients in Menoufia governorate, Egypt. MNF medical J ; 24:59-70.

22. El-Amin A, Obeid W, Abu-Aisha H. (2010): Renal Replacement Therapy in Sudan. Arab J Nephrol Transpl;3:31-6.

23. Shaheen FA, Al-Khader AA. (2005): Epidemiology and causes of end stage renal disease (ESRD). Saudi J Kidney Dis Transpl. July-September; 16(3):27781.

24. El Minshawy O, Ghabrah T., El Bassuoni E. (2014): End-stage Renal Disease in Tabuk Area, Saudi Arabia:An Epidemiological Study Saudi J Kidney Dis Transpl ;25(1):192-195.

25. Naicker S. (2009) : End-stage renal disease in sub-Saharan Africa. Ethn Dis. spring; 19 (1 Suppl 1):S1-13-5.

26. Collins AJ, Foley RN, Herzog C, et al.(2009): United States Renal Data System 2008 Annual Data Report. Am J Kidney Dis.;53:S1-374.

27. Malekmakan L, Haghpanah S, Pakfetrat M, et al(2009): Causes of chronic renal failure among Iranian hemodialysis patients. Saudi J Kidney Dis Transpl;20 (3):501-4.

28. Shigidi MM, Ramachandiran G, Rashed AH, Fituri OM. (2009):

29. Demographic data and hemodialysis population dynamics in Qatar: A five year survey. Saudi J Kidney Dis Transpl.; 20(3):493-500

30. Steenkamp R, Castledine C, Feest T, Fogarty D UK (2011): Renal Registry 13th Annual Report : Chapter 2: UK RRT prevalence in 2009: national and centre-specific analyses. Nephron ClinPract; 119 Suppl 2:c27-52.

31. Moukeh G, Yacoub R, Fahdi F, Rastam S, Albitar S.(2009): Epidemiology of hemodialysis patients in Aleppo city. Saudi J Kidney Dis Transpl. Jan; 20(1):140-6.

32. Batieha A, Abdallah S, Maghaireh M, Awad Z, Al-Akash N, et al (2007): Epidemiology and cost of haemodialysis in Jordan. East Mediterr Health J; 13:654-663.

33. Hussein M.M., Mooij J.M., Hegazy M.S., et al (2007): The impact of polymerase chain reaction assays for the detection of hepatitis C virus infection in hemodialysis units. Saudi J Kidney Dis. Transpl.;18:107-113.

34. Zahran AM (2014): HCV seroconversion in hemodialysis units ,Menoufia Medical Journal, 27:551-555 
35. 35-Kumar p. S., Venu G., Madhusudhana Rao A., et al (2011): Prevalence and Risk Factors of Hepatitis C Among Maintenance Hemodialysis Patients At A Tertiary-Care Hospital In Coimbatore, India. Journal of Clinical and Diagnostic Research; 5:725-728.

36. 36- Okuda K. And Hayashi H.(1996): Hepatitis $C$ virus infection among maintenance hemodialysis patients: A preventable problem of the world. Saudi J Gastroenterol,; 2:1-7.

37. 37- Selm S.B.(2010): Prevalence of hepatitis $C$ virus infection among hemodialysis patients ill a single center in Yemen. Saudi J Kidney Dis Transpl,; 21:1165-8. 